\title{
Avaliação do tratamento das dislipidemias em pacientes acompanhados no ambulatório de cardiologia do Hospital Universitário Walter Cantídio
}

\section{Evaluation of the treatment of dislipidemias in patients accompanied in the ambulatory of cardiology of the University Hospital Walter} Cantídio

\author{
Marcelo Caetano Pessoa Moreira ${ }^{1}$. Ricardo Pereira Silva ${ }^{1}$. \\ 1 Faculdade de Medicina, Universidade Federal do Ceará (UFC), Fortaleza, Ceará, Brasil.
}

\section{RESUMO}

Objetivos: realizar uma apreciação para o atingimento das metas lipídicas preconizadas na $\mathrm{V}$ Diretriz brasileira de dislipidemias e prevenção de aterosclerose. Metodologia: Realizou-se então uma pesquisa do tipo quantitativa com um estudo transversal retrospectivo feito por meio de uma coleta de dados nos prontuários de 148 pacientes acompanhados no ambulatório de cardiologia do Hospital Universitário Walter Cantídio (HUWC). Foram excluídos 90 prontuários devido à ausência de algum dado relevante para a análise estatística. Resultados: A análise estatística evidenciou que uma grande parcela dos pacientes acompanhados tinham níveis desejáveis $(24,1 \%)$ ou ótimos $(63,7 \%)$ de controle de LDL-c conforme o risco cardiovascular de cada indivíduo de acordo com a V Diretriz Brasileira de Dislipidemias e Prevenção de Aterosclerose da Sociedade Brasileira de Cardiologia. Considerando o conhecimento já estabelecido sobre a relação positiva entre os níveis de LDL-c e o risco de doença arterial coronariana, apenas uma minoria dos pacientes $(5,1 \%)$ deste estudo acompanhados neste serviço encontravam-se sob risco elevado de eventos cardiovasculares adversos. Conclusão: Os resultados obtidos permitiram concluir que o diagnóstico precoce e o tratamento adequado dos pacientes com dislipidemias são tangíveis. E para tal, reforçamos a necessidade da participação do Estado em políticas públicas direcionadas para assegurar o tratamento integral dos pacientes de baixa renda.

Palavras-chave: Dislipidemias. Hipercolesterolemia. Doenças cardiovasculares.

\begin{abstract}
Objective: This article aims to evaluate for achieving lipid targets preconized on the V Brazilian guideline for dyslipidemia and prevention of atherosclerosis. Methods: A quantitative research was performed with a retrospective cross-sectional study by collecting data from medical records of 148 assessed patients in the ambulatory at the Hospital Universitário Walter Cantídio (HUWC). 90 medical records were excluded due to the absence of relevant data to the statistical analysis. Results: The analysis showed that a great number of the assessed patients had desirable $(24,1 \%)$ or great $(63,7 \%)$ levels of control over the LDL-c levels, according to the $\mathrm{V}$ Brazilian guideline for dyslipidemia and prevention of atherosclerosis. And, considering the previously established knowledge about the positive relation between the LDL-c levels and the risk of a coronary disease, only few assessed $(5,1 \%)$ by this service were found under a high risk of adverse cardiovascular events. Conclusion: The obtained results allowed to conclude that early diagnosis and proper treatment to patients who have dyslipidemia are tangible. And, for such achievements, it is relevant to reinforce the need of a state participation with public policies towards the assurance of an integral treatment for low-income patients.
\end{abstract}

Keywords: Dyslipidemias. Hypercholesterolemia. Cardiovascular diseases.

Autor correspondente: Marcelo Caetano Pessoa Moreira, Rua Dr. Francisco Gadelha, 820, Luciano Cavalcante, Fortaleza, Ceará. CEP: $60811-120$. Telefone: +55 88 99633-1000. E-mail: marcelocaetanopessoa@gmail.com

Conflito de interesses: Não há qualquer conflito de interesses por parte de qualquer um dos autores.

Recebido em: 11 Fev 2019; Revisado em: 11 Ago 2019; Aceito em: 30 Dez 2019. 


\section{INTRODUÇÃO}

As dislipidemias são comorbidades relacionadas aos distúrbios do metabolismo lipídico repercutindo nas concentrações séricas dos diferentes componentes. É uma condição frequente em nosso meio com grande impacto na taxa de mortalidade cardiovascular por provocarem o desenvolvimento de doença arterial coronariana. ${ }^{1,2}$

As dislipidemias relacionadas a níveis elevados de colesterol total, triglicérides e lipoproteínas plasmáticas estão diretamente associadas à gênese e à evolução da aterosclerose. ${ }^{3}$ Sabe-se que a aterosclerose é uma doença inflamatória crônica multifatorial correlacionada a uma resposta sobre uma agressão endotelial. ${ }^{3}$

Concentrações plasmáticas elevadas de lipoproteínas de baixa densidade ligada ao colesterol (LDL-c), bem como concentrações plasmáticas reduzidas de HDL-c, têm sido consideradas fatores de risco independentes para o desenvolvimento da aterosclerose. ${ }^{3}$

Muito embora a utilização de outras lipoproteínas como análise do risco cardiovascular pareça óbvio, na prática clínica, apenas o colesterol total e LDL-c são tradicionalmente empregados como medidas na avaliação deste risco, pois a utilização destes já está embasada em diversos estudos e constituem o alvo terapêutico para a prevenção da doença cardiovascular. ${ }^{1}$

A dislipidemia ainda é relativamente pouco diagnosticada e tratada no sistema público de saúde do Brasil. Estudos mostraram que apenas um terço dos pacientes com doença arterial coronariana (DAC) confirmada estão adequadamente tratados e orientados quanto à importância dos riscos da dislipidemia. ${ }^{1,4}$

Diante deste panorama, podemos inferir que a prevenção primária da população assistida pelo sistema público de saúde está bem aquém do desejado pelos especialistas no assunto.

As dificuldades com o diagnóstico, uma baixa adesão, o tempo reduzido das consultas e o custo no tratamento estão entre os fatores que mais levam ao tratamento inadequado das dislipidemias. ${ }^{5}$

A necessidade de um aprofundamento sobre esses e outros vieses no acompanhamento e tratamento dos pacientes sabidamente com DAC possibilita uma orientação racional quanto à elaboração de estratégias específicas à adequação dos níveis séricos de lipídeos.

O objetivo geral do estudo é avaliar o tratamento das dislipidemias dos pacientes atendidos no ambulatório de cardiologia no Hospital Universitário Walter Cantídio (HUWC).

\section{MATERIAL E MÉTODOS}

Realizou-se uma pesquisa do tipo quantitativa com um estudo transversal retrospectivo feito por meio de uma coleta de dados nos prontuários de pacientes acompanhados no ambulatório de cardiologia do Hospital Universitário Walter Cantídio (HUWC) após aprovação do projeto pelo comitê de ética em pesquisa da Universidade Federal do Ceará (UFC). A fim de viabilizar a correta análise dos resultados, foi elaborado um formulário básico para a coleta dos dados.

A pesquisa contou com informações obtidas no banco de dados do sistema Tableau ${ }^{\circledR}$ de acompanhamento dos pacientes do ambulatório de cardiologia do Hospital Universitário Walter Cantídio para a seleção dos prontuários analisados. Foram selecionados os pacientes registrados no sistema Tableau ${ }^{\circledR}$ de 01/03/2018 até 31/07/2018 com idade maior ou igual a 20 anos e que estivessem dentro dos critérios preconizados pela Sociedade Brasileira de Cardiologia para indivíduos com muito alto risco cardiovascular. Para tal propósito, foram selecionados indivíduos com doença arterial coronariana diagnosticada e/ou acidente vascular encefálico e/ou doença arterial obstrutiva periférica com ou sem eventos clínicos prévios e/ou presença de obstrução maior ou igual a 50\% em qualquer território arterial avaliado através de ultrassonografia arterial com DOPPLER de artérias carótidas e vertebrais. Os indivíduos diagnosticados com dislipidemia tratada ou não também foram incluídos nesta pesquisa.

Contando com auxílio do Serviço de Arquivo Médico e Estatística (SAME) do HUWC, foram coletados dados relacionados aos fatores de risco para doença cardiovascular, além do nome, idade, número do prontuário, sexo, medicamentos em uso, data e resultado dos últimos exames laboratoriais de interesse coletados, a saber: colesterol total, HDL-c, LDL-c e triglicérides, no período de maio a julho de 2018.

Foram analisados os dados de 148 pacientes acompanhados no ambulatório de cardiologia no Hospital Universitário Walter Cantídio. Consideraram-se elegíveis os pacientes com idade maior ou igual a 20 anos, de ambos os sexos que estivessem ou não em tratamento de dislipidemia com algum tipo de hipolipemiante da classe dos inibidores da $\mathrm{HMG}-\mathrm{CoA}$ redutase, também conhecida como estatinas, e com alto risco para eventos cardiovasculares. Foram excluídos os prontuários com ausência de algum dado relevante para a análise estatística. As recomendações aconselhadas pela V Diretriz Brasileira de Dislipidemias e Prevenção de Aterosclerose ${ }^{1}$ e sua atualização de agosto de $2017^{3}$ da Sociedade Brasileira de Cardiologia foram utilizadas com o fim de se avaliar o quão ajustado estão os pacientes em relação às metas preconizadas por essa diretriz.

\section{RESULTADOS}

Nesta pesquisa, foram analisados os dados de 148 prontuários de pacientes acompanhados no ambulatório de cardiologia do HUWC. Destes, 90 prontuários foram excluídos por não se adequarem aos critérios de seleção da pesquisa ou por não dispor de todos os dados relevantes para a análise, como por exemplo, ausência de informações detalhadas sobre o perfil lipídico do paciente. A distribuição destes pacientes conforme o sexo foi de 32 indivíduos do sexo feminino $(55,1 \%)$ havendo, portanto, uma leve superioridade do sexo feminino. 
Pode-se observar que a grande maioria $(79,3 \%)$ era portadora de doença arterial coronariana e somente $10,3 \%$ dos pacientes haviam sofrido acidente vascular encefálico conforme análise das comorbidades estabelecidas na categoria de muito alto risco para eventos cardiovasculares (Gráficos 01 e 02).

A maioria dos pacientes da pesquisa encontrava-se na faixa etária de idosos (77,5\%) (Gráfico 03), segundo a Organização Mundial de Saúde (OMS). ${ }^{4}$

Gráfico 1. Prevalência de Doença Arterial Coronariana.

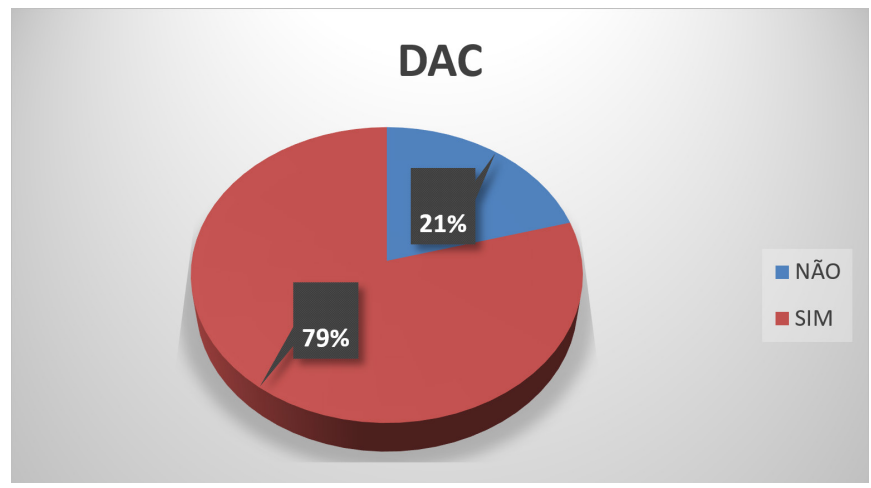

Gráfico 2. Prevalência de Acidente Vascular Cerebral.

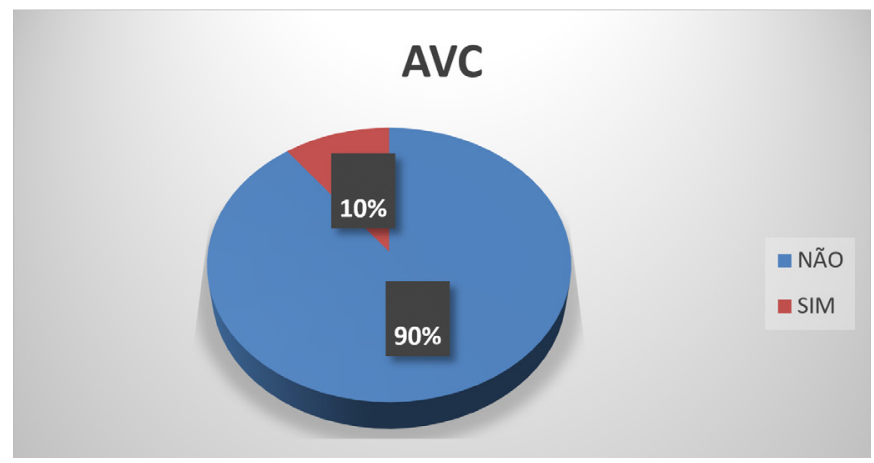

Gráfico 3. Indivíduos distribuídos por faixas etárias.

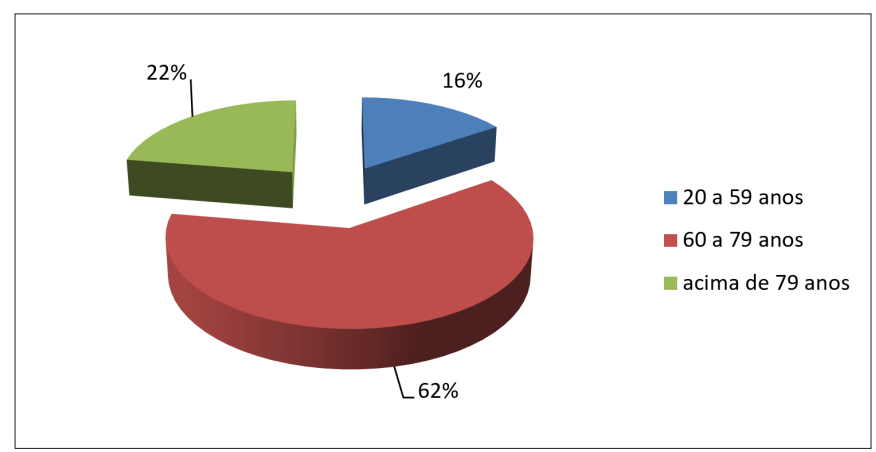

Todos os pacientes da pesquisa faziam uso regular de estatinas para controle lipídico, sendo a sinvastatina a de maior prevalência $(79,3 \%)$.

Uma análise pormenorizada dos subgrupos dos homens e das mulheres evidenciou uma semelhança de prevalência entre as comorbidades envolvidas na pesquisa, sendo a DAC a de maior prevalência em cada subgrupo (Gráfico 04). Assim também foi observado na análise do nível de LDL. A prevalência de pacientes com níveis ótimos de LDL foi de $63,7 \%$ com discreta superioridade para o subgrupo dos homens $(65,3 \%)$ de acordo com a V Diretriz Brasileira de Dislipidemias e Prevenção de Aterosclerose (Gráfico 05).

Uma subanálise das faixas etárias mostrou que pacientes masculinos do subgrupo de 20 a 59 anos acumulavam um número maior de comorbidades em comparação com do grupo dos octogenários, embora neste houvesse comparativamente um número maior de indivíduos. Entretanto, o subgrupo de 60 a 79 anos deteve o maior acúmulo de comorbidades entre todos os grupos.

Gráfico 4. Prevalência de Doença Arterial Coronariana entre os sexos.

DAC

Gráfico 5. Percentual dos níveis de LDL entre homens e mulheres da amostra segundo a V Diretriz Brasileira de Dislipidemias e Prevenção de Aterosclerose da Sociedade Brasileira de Cardiologia.

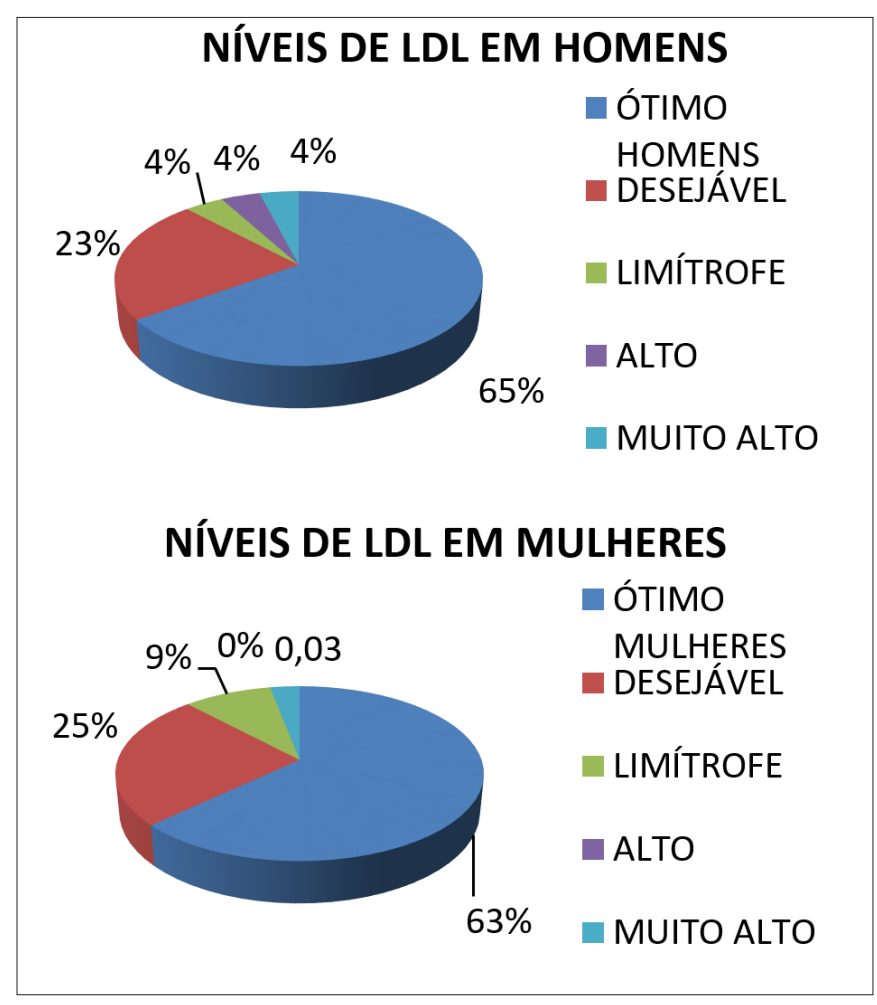




\section{DISCUSSÃO}

Trabalhos realizados em diferentes tipos de populações observaram alterações dos índices lipídicos segundo fatores sócios demográficos. ${ }^{6} \mathrm{O}$ presente trabalho teve por objetivo traçar uma análise sobre o tratamento da dislipidemia nos pacientes acompanhados no ambulatório de cardiologia do Hospital Universitário Walter Cantídio da Universidade Federal do Ceará.

Os resultados encontrados neste trabalho foram divergentes dos encontrados na literatura pesquisada. A análise dos resultados mostrou diferenças entre estudos realizados em outros grandes centros de pesquisa inclusive em países mais desenvolvidos. ${ }^{7}$ Os dados alcançados por essa pesquisa indicaram que muitos dos indivíduos envolvidos possuíam taxas consideradas ótimas de LDL-c e que $87,9 \%$ do total encontravam-se dentro dos limites preconizados para controle adequado de LDL-c, ou seja, entre os níveis desejável e ótimo de LDL-c.

Os resultados deste estudo revelaram uma taxa aceitável de dislipidemia controlada entre os participantes. Observouse que a minoria apresentou níveis muito altos de LDL-c, com igual prevalência entre os sexos. Tais achados estão em desacordo com outros estudos realizados em diferentes populações segundo FAGHERAZZI e col. (2008). ${ }^{2}$ Este autor realizou um estudo com 30 pacientes sabidamente cardiopatas ou com outros fatores de risco cardiovasculares e observou que todos possuíam taxas elevadas de LDL-c. ${ }^{2}$

VACANTI e colaboradores (2005) fizeram uma pesquisa com 190 pacientes com baixa renda e idade média de 70 anos, além de alto risco para doença cardiovascular, e viu que apenas $30 \%$ destes pacientes estavam dentro das metas lipídicas. ${ }^{5}$

A dificuldade em se atingir as metas preconizadas também ocorreu em outros grandes centros populacionais. A exemplo disso, o estudo L-TAP, realizado nos Estados Unidos da América contando com 4.888 indivíduos, que apontou que apenas 37\% dos pacientes estudados possuíam níveis desejáveis de LDL-c. ${ }^{8}$

Levando em consideração a baixa renda vivenciada e a mudança do estilo de vida a que os pacientes deveriam se submeter, VACANTI e colaboradores (2005) chegaram à conclusão que possivelmente a baixa adesão ao tratamento seria o principal motivo pelo qual os pacientes não atingiam as metas preconizadas. ${ }^{5}$ Em suma, a baixa renda da população estudada dificultou o acesso ao tratamento medicamentoso e a mudança para um estilo de vida mais saudável. ${ }^{4}$

Supomos que a valorização do serviço por parte dos pacientes e a orientação sobre as complicações de um descontrole lipídico durante $\mathrm{o}$ atendimento foram fatores importantes para motivar os pacientes a adotarem uma postura responsável quanto ao tratamento e adoção de um estilo de vida saudável, levando ao alcance das metas lipídicas preconizadas para proteção cardiovascular.

Entre as limitações observadas no presente estudo podemos destacar a baixa representatividade e a homogeneidade da amostra tida como de baixa renda e com limitado acesso a um tratamento preconizado pela V Diretriz Brasileira de Dislipidemias e Prevenção de Aterosclerose, além de várias perdas devidas a falta de informações nos prontuários médicos. Tais circunstâncias podem dar ao estudo um limitado poder estatístico.

Muito embora a elaboração da pesquisa trouxesse limitações de ordem logística e social, consideramos que a importância da mesma está em suscitar o interesse de profissionais da saúde na promoção de novas pesquisas nessa área e na motivação dos governantes em melhorar o acesso ao tratamento dos indivíduos com dislipidemias.

Apesar das limitações expostas, a condução das diferentes etapas da pesquisa foi realizada com elevado rigor metodológico que por sua vez implica em ausência de confusão ou vícios de seleção.

Considerando-se a elevada taxa de morbimortalidade cardiovascular dos pacientes com dislipidemia e o conhecimento já estabelecido da relação positiva entre os níveis de LDL-c e o risco de doença arterial coronariana, reforçamos a importância do diagnóstico e treinamento adequado dos profissionais que lidam diretamente com esse tipo de população.

\section{CONCLUSÃO}

Foi identificado um bom controle no perfil lipídico dos pacientes acompanhados no ambulatório, considerando as recomendações da V Diretriz Brasileira de Dislipidemias e Prevenção de Aterosclerose. Diante desses resultados, concluímos que o tratamento medicamentoso racional visando o alcance individual das metas preconizadas pode contribuir para a redução da prevalência de dislipidemias na população. Os benefícios destas medidas certamente se sobrepõem às dificuldades encontradas pelos pacientes ao tratamento, se considerarmos as consequências catastróficas de um evento cardiovascular isquêmico.

Enfatizamos que o incremento de políticas públicas com atenção aos pacientes dislipidêmicos de alto risco cardiovascular em seus diversos âmbitos certamente terá um papel importante na redução da morbimortalidade diante de um público progressivamente maior.

\section{REFERÊNCIAS}

1. Xavier HT, Izar MC, Faria J Neto, Assad MH, Rocha VZ, Sposito AC, et al. V Diretriz brasileira de dislipidemias e prevenção de aterosclerose. Arq Bras Cardiol. 2013;101(4, supl.1):1-30.
2. Fagherazzi S, Dias RL, Bortolon F. Impacto do exercício físico isolado e combinado com dieta sobre os níveis séricos de HDL, LDL, colesterol total e triglicerídeos. Rev Bras Med Esporte. 2008;(14)4:381-6. 
3. Faludi AA, Izar MC, Saraiva JF, Chacra AP, Bianco HT, Afiune A Neto. Atualização da diretriz brasileira de dislipidemia e prevenção de aterosclerose (2017). Arq Bras Cardio. 2017;(109)2:1-92.

4. Mendis S, Puska P, Norrving B. Global atlas oncardiovascular disease prevention and control. Genebra: World HealthOrganization; 2011. Capítulo 39, CVD prevention and control: why it should notbe ignored any longer?; p. 110-3.

5. Vacanti LJ, Santos SC, Fujita AM, Lima DS, Lopes AF, Vetoraz $\mathrm{R}$, et al. A baixa taxa de obtenção da meta do LDL-colesterol numa população de baixa renda. Arq Bras Cardiol. 2005;85(3):162-5.
6. Moraes SA, Checcio MV, Freitas ICM. Dislipidemia e fatores associados em adultos residentes em Ribeirão Preto, SP. Resultados do Projeto EPIDCV. Arq Bras Endocrinol Metab. 2013;57(9):691-701.

7. Ford ES, Li C, Pearson WS, Zhao G, Mokdad AH. Trends in hypercholesterolemia, treatment and control among united states adults. Int J Cardiol. 2010;140(2):226-35.

8. Pearson TA, Laurora I, Chu H, Kafonek S. The lipid treatment assessment project (L-TAP): a multicenter survey to evaluate the percentages of dyslipidemic patients receiving lipid-lowering therapy and achieving low-density lipoprotein cholesterol goals. Arch Intern Med. 2000;160(4):459-67.

\section{Como citar:}

Moreira MC, Silva RP. Avaliação do tratamento das dislipidemias em pacientes acompanhados no ambulatório de cardiologia do Hospital Universitário Walter Cantídio. Rev Med UFC. 2020 jul-set;60(3):19-23. 